\title{
INVESTIGATION OF POLARIZATION PHASE DIFFERENCE RELATED TO FOREST FIELDS CHARACTERIZATIONS
}

\author{
M. Majidi ${ }^{\text {a }}$, Y. Maghsoudi ${ }^{b}$ \\ a Faculty of Geodesy and Geomatics Engineering, K. N. Toosi University of Technology, Valiasr street, Tehran, IRAN - \\ (miladmajidi)@mail.kntu.ac.ir \\ b Faculty of Geodesy and Geomatics Engineering, K. N. Toosi University of Technology, Valiasr street, Tehran, IRAN \\ - (ymaghsoudi)@kntu.ac.ir
}

KEY WORDS: SAR, PPD, Biophysical Parameters, Forest Fields, Statistics

\begin{abstract}
:
The information content of Synthetic Aperture Radar (SAR) data significantly included in the radiometric polarization channels, hence polarimetric SAR data should be analyzed in relation with target structure. The importance of the phase difference between two co-polarized scattered signals due to the possible association between the biophysical parameters and the measured Polarization Phase Difference (PPD) statistics of the backscattered signal recorded components has been recognized in geophysical remote sensing.

This paper examines two Radarsat-2 images statistics of the phase difference to describe the feasibility of relationship with the physical properties of scattering targets and tries to understand relevance of PPD statistics with various types of forest fields. As well as variation of incidence angle due to affecting on PPD statistics is investigated.

The experimental forest pieces that are used in this research are characterized white pine (Pinus strobus L.), red pine (Pinus resinosa Ait.), jack pine (Pinus banksiana Lamb.), white spruce (Picea glauca (Moench Voss), black spruce (Picea mariana (Mill) B.S.P.), poplar (Populus L.), red oak (Quercus rubra L.), aspen and ground vegetation.

The experimental results show that despite of biophysical parameters have a wide diversity, PPD statistics are almost the same. Forest fields distributions as distributed targets have close to zero means regardless of the incidence angle. Also, The PPD distribution are function of both target and sensor parameters, but for more appropriate examination related to PPD statistics the observations should made in the leaf-off season or in bands with lower frequencies.
\end{abstract}

\section{INTRODUCTION}

Forest mapping have persuasively demonstrated that polarimetric synthetic aperture radar (PolSAR) measurements can be very helpful for classification, target detection and scene properties demodulation tasks. Actually SAR data are not only independent of the weather conditions, but also sensitive to the geometry of both the canopy and branching structure depending on the radar frequency. The Polarization Phase Difference (PPD) statistics of polarimetric radar that can be derived besides backscattering data is found to be an interesting parameter for the study of vegetation (Ulaby, 1987). Subsequently these studies extended for orchard fields (Wang, 1990). This is due to the possibility that a strong dependence between the biophysical parameters and the measured phase difference statistics of the backscattered electric field components exists. For the first time the single-look co-polarized phase difference pdf derived as a function of one parameter: the amplitude of the correlation coefficient of the two scattered field components (Eom, 1988). This was following generalized to include a second parameter: the phase of the correlation coefficient (Touzi, 1991). As well as experimental and theoretical studies were conducted to understanding the relationship between the rough surface characteristics and the phase difference distribution (Sarabandi, 1991). A multi-look co-polarized phase difference pdf was later derived (Lopes, 1992). Then both co- and cross-polarized phase difference pdfs have been derived in terms of the Mueller matrix elements and again, the co-polarized pdf is shown to be completely specified by two parameters: the degree of correlation and the polarized phase difference (Sarabandi, 1992). In the present study, we have used polarimetric data acquired at C-band with a view to study the statistics of the phase difference of the signals corresponding to a given pixel, or to a collection of pixels to describe the feasibility of relationship with the physical properties of scattering targets. One more aspect studied in this paper is the effect of incidence angle on phase difference statistics related to scene characterizations.

\section{METHODOLOGIES}

\subsection{Data and Site Description}

In this letter the Petawawa Research Forest (PRF) located near Chalk River, Ontario is selected as study site. It is nearly 200 $\mathrm{km}$ west of Ottawa and $180 \mathrm{~km}$ East of North Bay, Ontario, Canada. The PRF is the oldest ceaselessly operated forest research center in Canada and contains both boreal and temperate forest species. The elevation ranges from $140-280 \mathrm{~m}$ above sea level. Climate is continental with winter temperatures cold enough to support a fixed period of snow cover. The precipitation is evenly distributed through the year with 600 $\mathrm{mm}$ in the form of rain and $200 \mathrm{~mm}$ in the form of snow. January is the coldest month with a mean temperature of $12.4^{\circ} \mathrm{C}$ and July is the warmest with a mean temperature of $19.1^{\circ} \mathrm{C}$. It maintains more than 2000 experimental plots and 
sites making it an excellent resource for advanced remote sensing technologies.

The experimental forest pieces that are used in this research are nearly $484 \mathrm{~km}^{2}$ in size and are characterized white pine (Pinus strobus L.), red pine (Pinus resinosa Ait.), jack pine (Pinus banksiana Lamb.), white spruce (Picea glauca (Moench Voss), black spruce (Picea mariana (Mill) B.S.P.), poplar (Populus L.), red oak (Quercus rubra L.), aspen and ground vegetation. Forest fields are divided into three general categories as hardwood, softwood and vegetation that red oak and poplar trees are components of hard wood. It is clear, ground vegetation is in vegetation category and the other fields are components of soft wood.

Two Radarsat-2 fine-mode quad descending images acquired in 16th September 2008 (FQ4) and 19th September 2008 (FQ18) were used in this study. The incidence angle ranges are $43.5^{\circ}$ $45.8^{\circ}$ for $\mathrm{FQ} 4$ and $45^{\circ}-53.1^{\circ}$ for FQ18. Monitoring of environmental conditions indicated that both images had some (low) moisture on the canopy and open ground vegetation at the time of acquisition. As well as ground truth data were collected from a circa 2002 forest inventory map, aerial photographic interpretation, Landsat ETM + images and field visits.

\subsection{Pre-Processing}

Before using the SAR data some considerations should be take into account. Though, speckle filtering is a recommend preprocessing step in almost SAR processing techniques, it probability affects the pixel values. On the other hand, since our experiments significantly related to phase information and speckle filtering has a big impact on phase information, especially in high entropy environments (Lopez-Martinez 2005), in this study, for exact examining PPD statistics speckle filtering ignored.

As the ground truth data are in the ground coordinate system, the SAR image data must be geocoded. The geo-referenced Radarsat-1 data used in another project (Maghsoudi, 2011) over the same area is employed in this paper.

\subsection{PPD}

The polarization phase difference, $\Delta \varphi$ for each pixel is calculated in the following way:

$$
\Delta \varphi=\varphi_{H H}-\varphi_{V V}
$$

Where $\varphi_{\mathrm{HH}}$ is the phase of a wave transmitted and received in horizontal polarization and $\varphi \mathrm{vv}$ is the phase of a wave transmitted and received in vertical polarization. Actually the quantity of interest is obtained from the following product:

$$
S_{H H^{\prime}} S_{V V}^{*}=A_{H H} A_{V V} e^{\amalg \varphi}
$$

Where $S_{H H}$ and $S_{V V}$ are the received $\mathrm{HH}$ and VV signal voltages for a given image pixel, $A_{H H}$ and $A_{W V}$ are the amplitudes of $S_{H H}$ and $S_{V V}$, respectively. The extreme values of the PPD which are equal to either zero or $\pm \pi$ correspond to deterministic odd and even bounce scatterers, e.g., scatterers with a predominant reflective mechanism (Perissin, 2007). The PPD value diverges as the contribution from the diffusive scattering increases (Evans, 1988). Unless a target is azimuthally unsymmetrical, its backscattering properties should be identical for $\mathrm{HH}$ and $\mathrm{VV}$ polarizations at normal incidence. Hence, PPD is expected to be almost $0^{\circ}$ at normal and near normal incidence for most distributed targets (Ulaby, 1987). As mentioned in previous section nine covered fields were selected to examine the PPD statistics. Most fields were comprised of about 500 pixels. Probability density function (Pdf) of PPD is analyzed for covered fields and the relationships between PPD statistics and biophysical parameters are examined.

\section{RESULTS}

First of all, without considering the forest fields, PDD statistics of first ten near-range azimuth rows of the images with different incidence angle are compared. Fig.1 and Fig.2 shows the PPD distribution $p(\Delta \varphi)$ for the FQ4 and FQ18 images, respectively. The mean values are, of course, near to zero because it was used as the zero reference for the whole images.

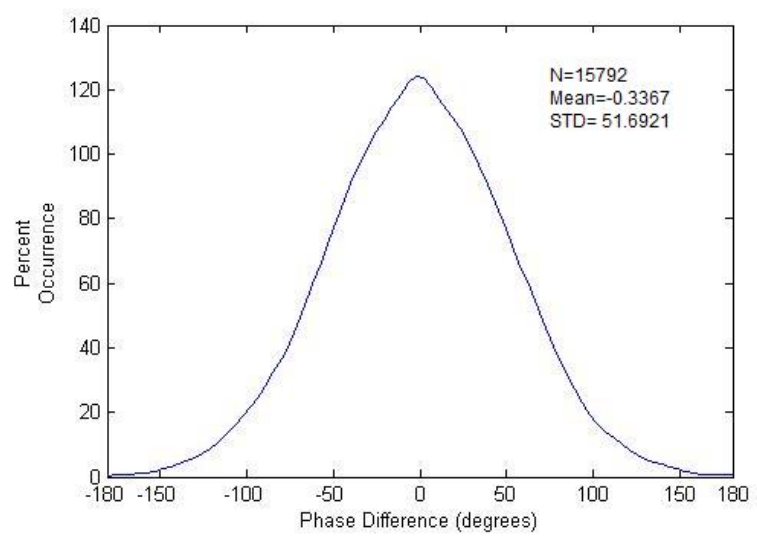

Figure 1. PPD distribution for the near-range of FQ4 The standard deviations $\sigma_{\varphi}$ that represents the variability in $\varphi$ assignable to the permanence of the measurement system, for two images is almost the same.

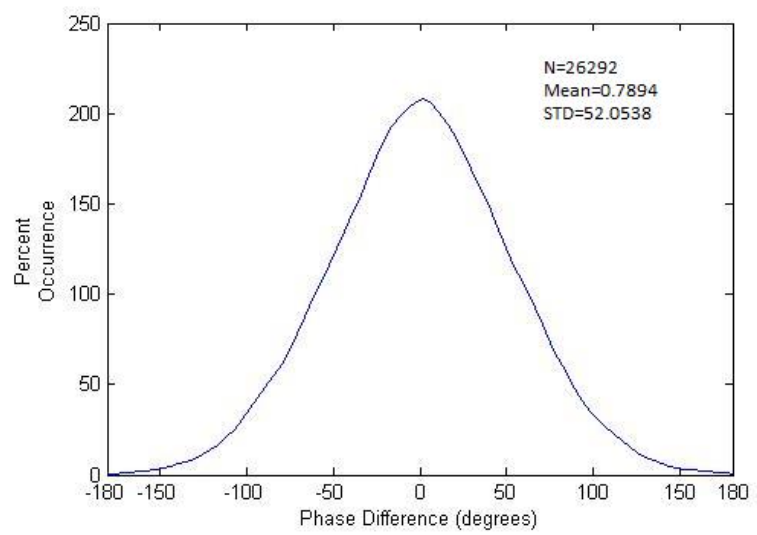

Figure 2. PPD distribution for the near-range of FQ18 Fig. 3 and Fig. 4 shows PPD distributions for two hardwood fields. Red oak, this deciduous tree grows straight and often reaching $20-30 \mathrm{~m}$ tall with a trunk of up to $50-100 \mathrm{~cm}$ diameter. Leaves are alternate, elliptic, 10-25 cm long and 8-15 $\mathrm{cm}$ wide (Keeler, Harriet L., 1900). As mentioned, another hardwood field in this study is Poplar. The genus of Poplar has a large genetic diversity, and can grow from anywhere between $15-50 \mathrm{~m}$ tall, with trunks of up to $2.5 \mathrm{~m}$ diameter. The leaves are spirally arranged, and vary in shape from triangular to circular or (rarely) lobed (Viereck L., 2007). 


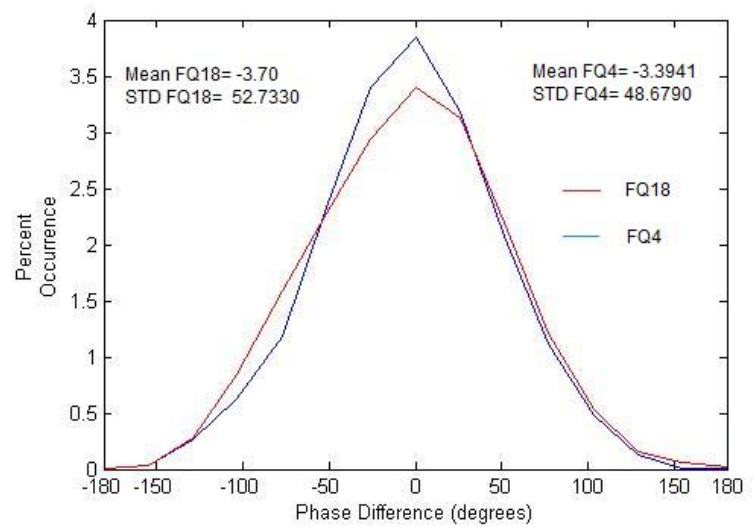

Figure 3. PPD distribution for Red oak

Both distributions have means close to zero, and their standard deviations are very similar together.

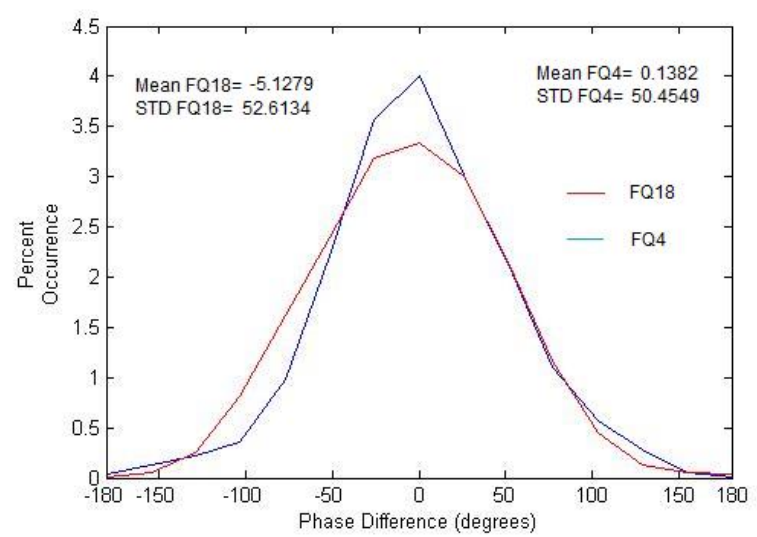

Figure 4. PPD distribution for Poplar

Fig. 5 shows PPD distributions for the white spruce. The White spruce is coniferous evergreen tree which grows normally to 15 to $30 \mathrm{~m}$ tall, but can grow up to $40 \mathrm{~m}$ tall with a trunk diameter of up to $1 \mathrm{~m}$. The crown is narrow - conic in young trees, becoming cylindrical in older trees. The leaves are needle-like, 12 to $20 \mathrm{~mm}$ long and rhombic in cross-section (Viereck L., 2007). PDD distributions have means close to zero, its standard deviations is close to reference images.

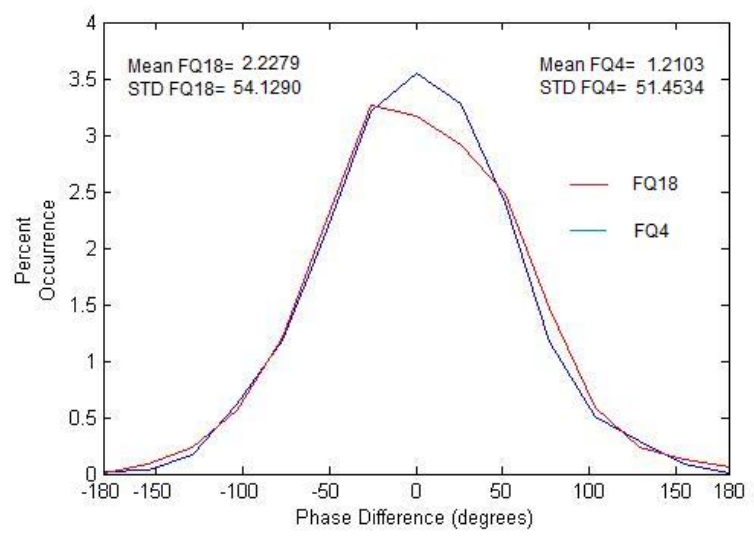

Figure 5. PPD distribution for White Spruce

In Fig. 6 White pine distributions is exhibited. The eastern White pine, has the distinction of being the tallest tree in eastern North America. The white pine normally grows to 35 to $50 \mathrm{~m}$ tall. Trunk Diameter of the white pines generally range from 1.0-1.6 m. In natural, the crown of this popular Christmas trees are conical shape with needle-like leaves (Ling, H. 2003). Despite of biophysical parameters have a wide diversity, PPD statistics are almost the same. Although white pine has some differences with reference, but it is so negligible that it could be dramatically overlook.

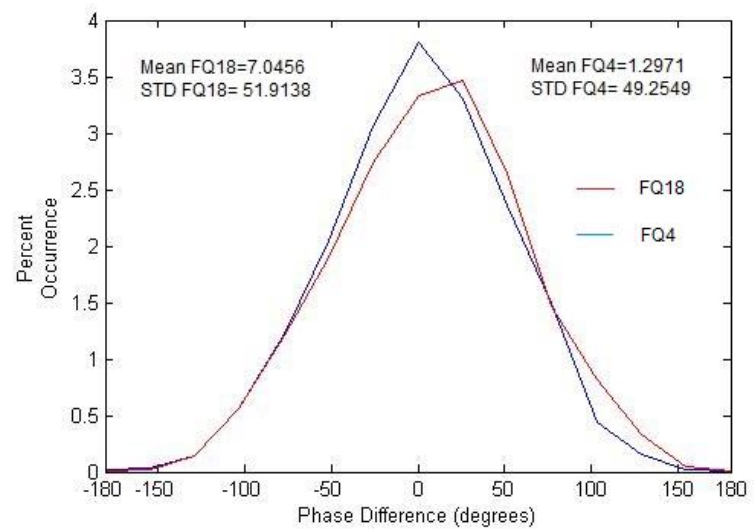

Figure 6. PPD distribution for White pine

Jack Pine ranges from 9-22 $\mathrm{m}$ in height. It's a smaller tree that can grow almost anywhere. The leaves are in fascicles of two, needle-like, twisted, slightly yellowish-green, and $2-4 \mathrm{~cm}$ long. Trunk is up to $60 \mathrm{~cm}$ in diameter (Keeler, Harriet L., 1900). Fig 7 shows distributions for Jack pine.

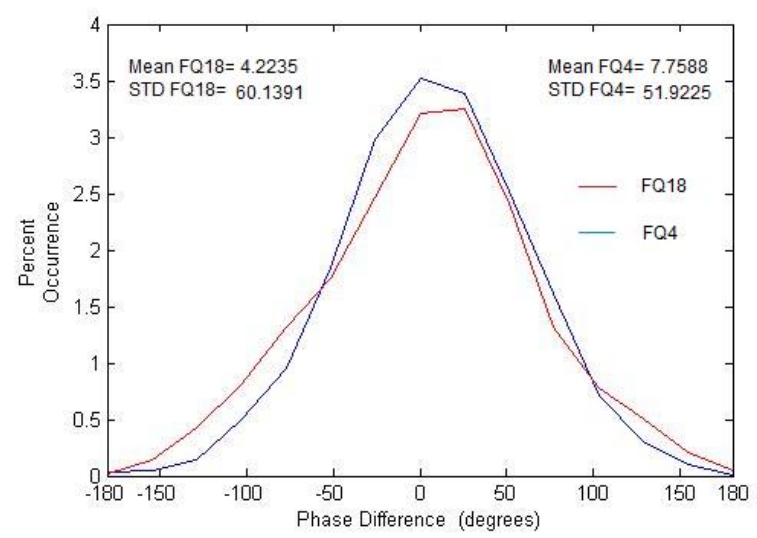

Figure 7. PPD distribution for Jack pine

According to relatively low height range of jack pine, bi-static reflection by the vertical stalks and propagation delay between the H-polarized and V-polarized waves as they travel through the canopy down to the soil surface and back (subsequent to specular reflections by the underlying surface and the stalk, in either order) let to remarkable standard deviation difference with reference image.

The ground vegetation sites consisted of grass, herbs, sweet fern, bracken and low shrubs of moderate or high density. It is true that the height range of ground vegetation sites in compare to the other fields are the lowest, but unlike the Jack pine, PPD statistics are the same with references. Because most of jack pine mass is contained in its vertical stalks, acts like a uniaxial crystal characterized by different velocities of propagation for waves with horizontal and vertical polarization. Thus, it is hypothesized that the observed backscatter is contributed by a combination of propagation delay, forward scatter by the underlying surface, and specular bi-static reflection by the stalks. Fig 8 shows distributions for the ground vegetation sites. The experiments is also done for another forest fields as aspen, black spruce and red pine. In all cases PDD distributions have means close to zero and their standard deviations are close to reference images. 


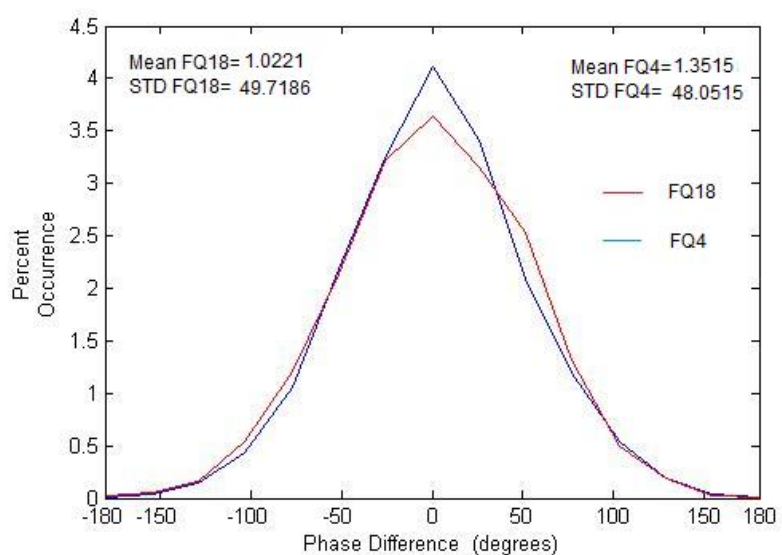

Figure 8. PPD distribution for ground vegetation

\section{CONCLUSION}

The results of observations discussed in the preceding section indicate that relative to the selected reference, namely the nearrange strip of the image, forest fields exhibit PPD distributions with means within -10 to +10 . It intends, distributions are close to zero means regardless of the incidence angle.

According to the definition of PPD, we note that in order to the phase difference to be different from zero, at least one of the following conditions should be satisfied: 1) The phase difference is caused by the scatterers in the target, 2) Phase difference is due to time delay resulting from the condition that the scatterers dominating the VV-polarized backscatter are at a range different from that for those dominating the $\mathrm{HH}$-polarized backscatter, or 3) PPD is due to time delay resulting from the condition that the phase velocity in the forest field canopy for $\mathrm{V}$ polarization is different from that for $\mathrm{H}$ polarization.

Whereas, in this study forest fields as distributed targets are examined, first and second conditions are hardly possible to achieve. Also according to the results, it seems the phase velocity for $\mathrm{V}$ polarization is almost the same with $\mathrm{H}$ polarization.

In one case, whereas most of jack pine mass is contained in its vertical stalks and also for the low height, PPD distributions let to remarkable standard deviation difference with reference images.

Special condition that needs to be satisfied in order for the polarization phase difference to be significantly different from zero is for the primary source of the backscattered signal to be the result of forward scattering by the underlying surface and the vertical stalks. This condition, requires that the canopy be a comparatively low-loss medium, which was satisfied in this case by the fact that the observations were made in the leaf-off season or in bands with lower frequencies.

\section{REFRENCES}

Eom H.J. and Boerner W.M., 1988. Statistical properties of phase difference between two orthogonally-polarized SAR signals, Proc. of IGARSS'88 Edinburgh Scotland, 1. pp. 65-66.

Evans D. L., Farr T. G., van Zyl J. J. and H. Zebker, 1988. Radar polarimetry: Analysis tools and applications, IEEE Trans. Geosci. Remote S, 26(6), pp. 748-789.

Keeler, Harriet L. 1900. Our Native Trees and How to Identify Them. New Roak: Charles Scriber's Sons. pp. 349-354.
Ling, H. 2003. The Eastern White Pine. Native Plant Society of NJ Newsletter. pp. 2-3.

Lopes A., Mougin E., Beaudoin A., Goze S., Nezry E., Touzi R., Karam M.A. and Fung A.K., 1992. Phase difference statistics related to sensor and forest parameters, Proc. Of IGARSS'92 Houston Texas, 1, pp.779-781.

Lopez-Martinez C., Pottier E. and Cloude S., 2005. Statistical assessment of eigenvector based target decomposition theorems in radar polarimetry. IEEE Trans. on Geosci and Remote S, 43(9), pp. 2058-2074.

Maghsoudi Y., Collins M. and Leckie D., 2011. Speckle reduction for the forest mapping analysis of multi-temporal radarsat-1 images. International Journal of Remote Sensing, 33(5), pp.1349-1359.

Perissin D. and Ferretti A., 2007. Urban-target recognition by means of repeated space borne SAR images. IEEE Trans. Geosci and Remote S., 45(12), pp. 4043-4058.

Sarabandi K., Oh Y. and Ulaby F.T., 1991. Polarimetric radar measurements of bare soil surfaces at microwave frequencies, IEEE Geosci and Remote S Symposium IGARSS '91, 2, pp. 387390.

Sarabandi K., 1992. Derivation of phase statistics from the Mueller matrix, Radio Science, 27(5), pp.553-560.

Touzi R. and Lopes A., 1991. Distribution of the Stokes parameters and the phase difference in polarimetric SAR data", Proc. of IGARSS'91 Helsinki Finland, 1, pp.103-106.

Ulaby F. T., Held D., Dobson M. C., McDonald K. and T. Senior, 1987. Relating polarization phase difference of SAR signals to scene properties. IEEE Trans. Geosci. Remote S., GRS-25(1), pp. 83-92.

Viereck L. Little E. L. 2007. Alaska Trees and Shrubs. Snowy Old Books. pp. 51-89.

Wang J.R. and MO T., 1990. Polarization phase difference of orchard trees, Int. J. Remote Sensing, 11, pp. 1255-1265. 